\title{
Outcomes Following Implementation of a Hospital-Wide, Multicomponent Delirium Care Pathway
}

\author{
Sara C LaHue, MD ${ }^{1,2 *}$, Judy Maselli, MSPH³, Stephanie Rogers, MD³, Julie Casatta, NP4, Jessica Chao, MBA, PharmD \\ Rhiannon Croci, BSN ${ }^{3}$, Ralph Gonzales, MD, MSPH ${ }^{3,5}$, Brian Holt, MHA ${ }^{6}$, S Andrew Josephson, MD ${ }^{1,2}, \mathrm{Sudha}_{\text {Lama, }} \mathrm{MS}^{3}$, \\ Catherine Lau, MD³, Charles McCulloch, PhD7, John C Newman, MD, PhD ${ }^{3,8}$, Mark Terrelonge, MD, MPH ${ }^{1,2}$, \\ Jan Yeager, MDes ${ }^{5}$, Vanja C Douglas, $\mathrm{MD}^{1,2}$
}

\begin{abstract}
${ }^{1}$ Department of Neurology, School of Medicine, University of California, San Francisco, California; ${ }^{2}$ Weill Institute for Neurosciences, Department of Neurology, University of California, San Francisco, California; ${ }^{3}$ Department of Medicine, School of Medicine, University of California, San Francisco, California; ${ }^{4}$ Department of Neurological Surgery, University of California, San Francisco, California; ${ }^{5}$ Clinical Innovation Center, University of California, San Francisco, California; ${ }^{6}$ Continuous Improvement Department, University of California, San Francisco, California; ${ }^{7}$ Epidemiology \& Biostatistics, University of California, San Francisco, California; ${ }^{8}$ Buck Institute for Research on Aging, Novato, California.
\end{abstract}

BACKGROUND: Delirium is associated with poor clinical outcomes that could be improved with targeted interventions.

OBJECTIVE: To determine whether a multicomponent delirium care pathway implemented across seven specialty nonintensive care units is associated with reduced hospital length of stay (LOS). Secondary objectives were reductions in total direct cost, odds of 30-day hospital readmission, and rates of safety attendant and restraint use.

METHODS: This retrospective cohort study included 22,708 hospitalized patients (11,018 preintervention) aged $\geq 50$ years encompassing seven nonintensive care units: neurosciences, medicine, cardiology, general and specialty surgery, hematology-oncology, and transplant. The multicomponent delirium care pathway included a nurse-administered delirium risk assessment at admission, nurse-administered delirium screening scale every shift, and a multicomponent delirium intervention. The primary study outcome was LOS for all units combined and the medicine unit separately. Secondary outcomes included total direct cost, odds of 30-day hospital readmission, and rates of safety attendant and restraint use.

RESULTS: Adjusted mean LOS for all units combined decreased by $2 \%$ post intervention (proportional change, $0.98 ; 95 \% \mathrm{Cl}, 0.96-0.99 ; P=.0087)$. Medicine unit adjusted LOS decreased by $9 \%$ (proportional change, $0.91 ; 95 \% \mathrm{Cl}, 0.83-0.99 ; P=.028$ ). For all units combined, adjusted odds of 30-day readmission decreased by $14 \%$ (odds ratio [OR], 0.86; 95\% Cl, 0.80-0.93; $P=.0002$ ). Medicine unit adjusted cost decreased by $7 \%$ (proportional change, 0.93; 95\% Cl, 0.89-0.96; $P=.0002$ ).

CONCLUSION: This multicomponent hospital-wide delirium care pathway intervention is associated with reduced hospital LOS, especially for patients on the medicine unit. Odds of 30-day readmission decreased throughout the entire cohort. Journal of Hospital Medicine 2021;16:397-403. (C) 2021 Society of Hospital Medicine

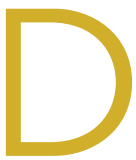
elirium is an acute disturbance in mental status characterized by fluctuations in cognition and attention that affects more than 2.6 million hospitalized older adults in the United States annually, a rate that is expected to increase as the population ages. ${ }^{1-4}$ Hospitalacquired delirium is associated with poor outcomes, including prolonged hospital length of stay (LOS), loss of independence, cognitive impairment, and even death. ${ }^{5-10}$ Individuals who develop delirium do poorly after hospital discharge and are more likely to be readmitted within 30 days. ${ }^{11}$ Approximately $30 \%$ to $40 \%$ of hospital-acquired delirium cases are preventable. ${ }^{10,12} \mathrm{Howev}$ er, programs designed to prevent delirium and associated com-

*Corresponding Author: Sara C LaHue, MD; Email: sara.lahue@ucsf.edu.

Published online first June 8, 2021.

Find additional supporting information in the online version of this article.

Received: September 6, 2020; Revised: January 14, 2021

Accepted: January 21, 2021

๑) 2021 Society of Hospital Medicine DOI 10.12788/jhm.3604 plications, such as increased LOS, have demonstrated variable success. ${ }^{12-14}$ Many studies are limited by small sample sizes, lack of generalizability to different hospitalized patient populations, poor adherence, or reliance on outside funding. 12,13,15-18

Delirium prevention programs face several challenges because delirium could be caused by a variety of risk factors and precipitants. ${ }^{19,20}$ Some risk factors that occur frequently among hospitalized patients can be mitigated, such as sensory impairment, immobility from physical restraints or urinary catheters, and polypharmacy. ${ }^{20,21}$ Effective delirium care pathways targeting these risk factors must be multifaceted, interdisciplinary, and interprofessional. Accurate risk assessment is critical to allocate resources to high-risk patients. Delirium affects patients in all medical and surgical disciplines, and often is underdiagnosed. ${ }^{19,22}$ Comprehensive screening is necessary to identify cases early and track outcomes, and educational efforts must reach all providers in the hospital. These challenges require a systematic, pragmatic approach to change. 
The purpose of this study was to evaluate the association between a delirium care pathway and clinical outcomes for hospitalized patients. We hypothesized that this program would be associated with reduced hospital LOS, with secondary benefits to hospitalization costs, odds of 30-day readmission, and delirium rates.

\section{METHODS}

\section{Study Design}

In this retrospective cohort study, we compared clinical outcomes the year before and after implementation of a delirium care pathway across seven hospital units. The study period spanned October 1, 2015, through February 28, 2019. The study was approved by the University of California, San Francisco Institutional Review Board (\#13-12500).

\section{Multicomponent Delirium Care Pathway}

The delirium care pathway was developed collaboratively among geriatrics, hospital medicine, neurology, anesthesiology, surgery, and psychiatry services, with an interprofessional team of physicians, nurses, pharmacists, and physical and occupational therapists. This pathway was implemented in units consecutively, approximately every 4 months in the following order: neurosciences, medicine, cardiology, general surgery, specialty surgery, hematology-oncology, and transplant. The same implementation education protocols were performed in each unit. The pathway consisted of several components targeting delirium prevention and management (Appendix Figure 1 and Appendix Figure 2). Systematic screening for delirium was introduced as part of the multicomponent intervention. Nursing staff assessed each patient's risk of developing delirium at admission using the AWOL score, a validated delirium prediction tool. ${ }^{23}$ AWOL consists of: patient Age, spelling "World" backwards correctly, Orientation, and assessment of iLIness severity by the nurse. For patients who spoke a language other than English, spelling of "world" backwards was translated to his or her primary language, or if this was not possible, the task was modified to serial 7s (subtracting 7 from 100 in a serial fashion). This modification has been validated for use in other languages. ${ }^{24}$ Patients at high risk for delirium based on an AWOL score $\geq 2$ received a multidisciplinary intervention with four components: (1) notifying the primary team by pager and electronic medical record (EMR), (2) a nurse-led, evidence-based, nonpharmacologic multicomponent intervention, ${ }^{25}$ (3) placement of a delirium order set by the physician, and (4) review of medications by the unit pharmacist who adjusted administration timing to occur during waking hours and placed a note in the EMR notifying the primary team of potentially deliriogenic medications. The delirium order set reinforced the nonpharmacologic multicomponent intervention through a nursing order, placed an automatic consult to occupational therapy, and included options to order physical therapy, order speech/language therapy, obtain vital signs three times daily with minimal night interruptions, remove an indwelling bladder catheter, and prescribe melatonin as a sleep aid.

The bedside nurse screened all patients for active delirium every 12-hour shift using the Nursing Delirium Screening Scale (NuDESC) and entered the results into the EMR. ${ }^{23,26}$ Capturing
NuDESC results in the EMR allowed communication across medical providers as well as monitoring of screening adherence. Each nurse received two in-person trainings in staff meetings and one-to-one instruction during the first week of implementation. All nurses were required to complete a 15-minute training module and had the option of completing an additional 1-hour continuing medical education module. If a patient was transferred to the intensive care unit (ICU), delirium was identified through use of the ICU-specific Confusion Assessment Method (CAM-ICU) assessments, which the bedside nurse performed each shift throughout the intervention period. ${ }^{27}$ Nurses were instructed to call the primary team physician after every positive screen. Before each unit's implementation start date, physicians with patients on that unit received education through a combination of grand rounds, resident lectures and seminars, and a pocket card on delirium evaluation and management.

\section{Participants and Eligibility Criteria}

We included all patients aged $\geq 50$ years hospitalized for $>1$ day on each hospital unit (Figure). We included adults aged $\geq 50$ years to maximize the number of participants for this study while also capturing a population at risk for delirium. Because the delirium care pathway was unit-based and the pathway was rolled out sequentially across units, only patients who were admitted to and discharged from the same unit were included to better isolate the effect of the pathway. Patients who were transferred to the ICU were only included if they were discharged from the original unit of admission. Only the first hospitalization was included for patients with multiple hospitalizations during the study period.

\section{Patient Characteristics}

Patient demographics and clinical data were collected after discharge through Clarity and Vizient electronic databases (Table 1 and Table 2). All Elixhauser comorbidities were included except for the following International Classification of Disease, Tenth Revision, Clinical Modification (ICD-10) codes that overlapped with a delirium diagnosis: G31.2, G93.89, G93.9, G94, R41.0, and R41.82 (Appendix Table 1). Severity of illness was obtained from Vizient, which calculates illness severity based on clinical and claims data (Appendix Table 1).

\section{Delirium Metrics}

Delirium screening was introduced as part of the multicomponent intervention, and therefore delirium rates before the intervention could not be determined. Trends in delirium prevalence and incidence after the intervention are reported. Prevalent delirium was defined as a single score of $\geq 2$ on the nurse-administered NuDESC or a positive CAM-ICU at any point during the hospital stay. Incident delirium was identified if the first NuDESC score was negative and any subsequent NuDESC or CAM-ICU score was positive.

\section{Outcomes}

The primary study outcome was hospital LOS across all participants. Secondary outcomes included total direct cost and 


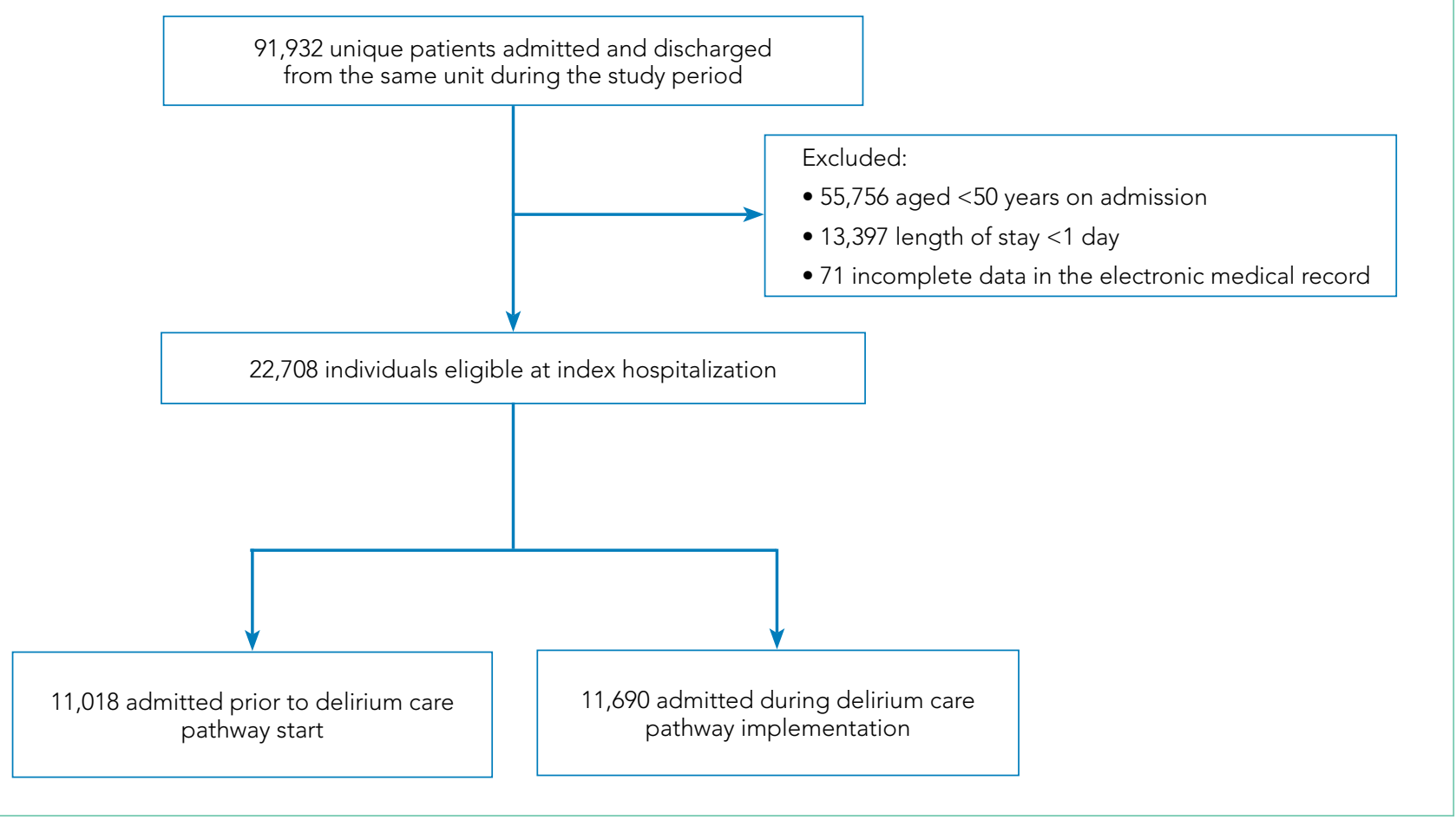

FIG. Flow Diagram of Study Participant Inclusion and Exclusion

odds of 30-day hospital readmission. Readmissions tracked as part of hospital quality reporting were obtained from Vizient and were not captured if they occurred at another hospital. We also examined rates of safety attendant and restraint use during the study period, defined as the number of safety attendant days or restraint days per 1,000 patient days.

Because previous studies have demonstrated the effectiveness of multicomponent delirium interventions among elderly general medical patients, ${ }^{12}$ we also investigated these same outcomes in the medicine unit alone.

\section{Statistical Analysis}

The date of intervention implementation was determined for each hospital unit, which was defined as time(0) [t(0)]. The 12-month postintervention period was divided into four 3 -month epochs to assess for trends. Data were aggregated across the seven units using $t(0)$ as the start date, agnostic to the calendar month. Demographic and clinical characteristics were collected for the 12-months before $t(0)$ and the four 3-month epochs after $t(0)$. Univariate analysis of outcome variables comparing trends across the same epochs were conducted in the same manner, except for the rate of delirium, which was measured after $\mathrm{t}(0)$ and therefore could not be compared with the preintervention period.

Multivariable models were adjusted for age, sex, race/ethnicity, admission category, Elixhauser comorbidities, severity of illness quartile, and number days spent in the ICU. Admission category referred to whether the admission was emergent, urgent, or elective/unknown. Because it took 3 months after $t(0)$ for each unit to reach a delirium screening compliance rate of $90 \%$, the intervention was only considered fully implemented after this period. A ramp-up variable was set to 0 for admissions occurring prior to the intervention to $t(0), 1 / 3$ for admissions occurring 1 month post intervention, $2 / 3$ for 2 months post intervention, and 1 for admissions occurring 3 to 12 months post intervention. In this way, the coefficient for the ramp-up variable estimated the postintervention versus preintervention effect. Numerical outcomes (LOS, cost) were log transformed to reduce skewness and analyzed using linear models. Coefficients were back-transformed to provide interpretations as proportional change in the median outcomes.

For LOS and readmission, we assessed secular trends by including admission date and admission date squared, in case the trend was nonlinear, as possible predictors; admission date was the specific date-not time from $t(0)$ - to account for secular trends and allow contemporaneous controls in the analysis. To be conservative, we retained secular terms (first considering the quadratic and then the linear) if $P<.10$. The categorical outcome (30-day readmission) was analyzed using a logistic model. Count variables (delirium, safety attendants, restraints) were analyzed using Poisson regression models with a log link, and coefficients were backtransformed to provide rate ratio interpretations. Because delirium was not measured before $\mathrm{t}(0)$, and because the intervention was considered to take 3 months to become fully effective, baseline delirium rates were defined as those in the first 3 months adjusted by the ramp-up variable. For each outcome we included hospital unit, a ramp-up variable 
TABLE 1. Participant Demographics and Clinical Characteristics for Preintervention Period (Epoch 0)

\begin{tabular}{lc}
\hline & $\begin{array}{r}\text { Epoch } 0:-12 \text { to } 0 \text { months } \\
(\mathbf{n}=11,018)\end{array}$ \\
\hline Hospital units, No. (\%) & $1,233(11.2)$ \\
Neurosciences & $2,552(23.2)$ \\
Medicine & $1,309(11.9)$ \\
Cardiology & $1,297(11.8)$ \\
Specialty surgery & $2,859(26.0)$ \\
General surgery & $649(5.9)$ \\
Hematology-oncology & $1,119(10.2)$ \\
Transplant
\end{tabular}

Age on admission, y

\begin{tabular}{cc} 
Mean (SD) & $66.7(10.8)$ \\
Median (IQR) & $65(58-73)$ \\
\hline Female, No. (\%) & $5,255(47.7)$ \\
\hline
\end{tabular}

Race/ethnicity, No. (\%)

$\begin{array}{lc}\text { White, non-Hispanic } & 6,261(56.8) \\ \text { Hispanic } & 1,161(10.5) \\ \text { Black } & 975(8.9) \\ \text { Asian } & 1,710(15.5) \\ \text { Other } & 911(8.3)\end{array}$

Admission category, No. (\%)

\begin{tabular}{lc} 
Emergent & $4,237(38.5)$ \\
Urgent & $2,285(20.7)$ \\
Routine/transplant/unknown & $4,496(40.8)$ \\
\hline Intensive care unit days, mean (SD) & $0.27(1.50)$
\end{tabular}

Abbreviation: IQR, interquartile range.

(measuring the pre- vs postintervention effect), and their interaction. If there was no statistically significant interaction, we presented the outcome for all units combined. If the interaction was statistically significant, we looked for consistency across units and reported results for all units combined when consistent, along with site-specific results. If the results were not consistent across the units, we provided site-specific results only. All statistical analyses were performed using SAS software, version 9.4 (SAS Institute Inc).

\section{RESULTS}

\section{Participant Demographics and Clinical Characteristics}

A total of 22,708 individuals were included in this study, with 11,018 in the preintervention period (Table 1 and Table 2). Most patients were cared for on the general surgery unit $(n=5,899)$, followed by the medicine unit $(n=4,923)$. The smallest number of patients were cared for on the hematology-oncology unit $(n=1,709)$. Across the five epochs, patients were of similar age and sex, and spent a similar number of days in the ICU. The population was diverse with regard to race and ethnicity; there were minor differences in admission category. There were also minor differences in severity of illness and some comorbidities between timepoints (Appendix Table 1).

\section{Delirium Metrics}

Delirium prevalence was $13.0 \%$ during the first epoch post intervention, followed by $12.0 \%, 11.7 \%$, and $13.0 \%$ in the subsequent epochs $(P=.91)$. Incident delirium occurred in $6.1 \%$ of patients during the first epoch post intervention, followed by $5.3 \%, 5.3 \%$, and $5.8 \%$ in the subsequent epochs $(P=.63)$.

\section{Primary Outcome}

Epoch-level data for LOS before and after the intervention is shown in Appendix Table 2. The mean unadjusted LOS for all units combined did not decrease after the intervention, but in the adjusted model, the mean LOS decreased by $2 \%$ after the intervention ( $P=.0087$; Table 3$)$.

\section{Secondary Outcomes}

The odds of 30-day readmission decreased by $14 \%(P=.0002)$ in the adjusted models for all units combined (Table 3). There was no statistically significant reduction in adjusted total direct hospitalization cost or rate of restraint use. The safety attendant results showed strong effect modification across sites; the site-specific estimates are provided in Appendix Table 3. However, the estimated values all showed reductions, and a number were large and statistically significant.

\section{Medicine Unit Outcomes}

On the medicine unit alone, we observed a statistically significant reduction in LOS of $9 \%$ after implementation of the delirium care pathway $(P=.028)$ in the adjusted model (Table 3$)$. There was an associated $7 \%$ proportional decrease in total direct cost $(P=.0002)$. Reductions in 30-day readmission and safety attendant use did not remain statistically significant in the adjusted models.

\section{DISCUSSION}

Implementation of a hospital-wide multicomponent delirium care pathway was associated with reduced hospital LOS and 30-day hospital readmission in a study of 22,708 hospitalized adults at a tertiary care, university hospital in Northern California, encompassing both medical and surgical acute care patients. When evaluating general medicine patients alone, pathway implementation was associated with reductions in LOS and total direct cost. The cost savings of $7 \%$ among medical patients translates to median savings of $\$ 1,237$ per hospitalization. This study_one of the largest to date examining implementation of a hospital-wide delirium care pathway-supports use of a multicomponent delirium care pathway for older adults hospitalized for a range of conditions.

Multicomponent pathways for delirium prevention and management are increasingly being used in hospital settings. 
TABLE 2. Participant Demographics and Clinical Characteristics for Postintervention Period (Epochs 1-4)

\begin{tabular}{|c|c|c|c|c|}
\hline & $\begin{array}{l}\text { Epoch 1: } 0 \text { to } 3 \text { months } \\
\qquad(\mathrm{n}=2,935)\end{array}$ & $\begin{array}{l}\text { Epoch 2: } 3 \text { to } 6 \text { months } \\
\qquad(\mathrm{n}=2,861)\end{array}$ & $\begin{array}{l}\text { Epoch 3: } 6 \text { to } 9 \text { months } \\
\qquad(\mathrm{n}=2,947)\end{array}$ & $\begin{array}{l}\text { Epoch 4: } 9 \text { to } 12 \text { months } \\
\qquad(\mathrm{n}=2,947)\end{array}$ \\
\hline \multicolumn{5}{|l|}{ Hospital units, No. (\%) } \\
\hline Medicine & $567(19.9)$ & $508(17.2)$ & $652(22.1)$ & $644(21.9)$ \\
\hline Cardiology & $270(9.5)$ & $323(11.0)$ & $325(11.0)$ & $316(10.7)$ \\
\hline Hematology-oncology & $182(6.4)$ & $380(12.9)$ & $267(9.1)$ & $231(7.8)$ \\
\hline Transplant & $317(11.1)$ & $285(9.7)$ & $327(11.1)$ & $340(11.5)$ \\
\hline \multicolumn{5}{|l|}{ Age on admission, y } \\
\hline Mean (SD) & $67(11.0)$ & $67(10.7)$ & $66.6(10.6)$ & $67.2(10.8)$ \\
\hline White, non-Hispanic & $1,560(54.8)$ & $1,656(56.2)$ & $1,626(55.2)$ & $1,660(56.3)$ \\
\hline Hispanic & $302(10.6)$ & $374(12.7)$ & $360(12.7)$ & $347(11.8)$ \\
\hline Black & $308(10.8)$ & $246(8.4)$ & $28(9.5)$ & $249(8.5)$ \\
\hline Asian & $454(15.9)$ & $452(15.3)$ & $438(14.9)$ & $464(15.7)$ \\
\hline Other & $225(7.9)$ & $219(7.4)$ & $243(8.3)$ & $227(7.7)$ \\
\hline \multicolumn{5}{|l|}{ Admission category, №. (\%) } \\
\hline Emergent & $1,116(39.2)$ & $1,151(39.1)$ & $1,235(41.9)$ & $1,170(39.7)$ \\
\hline Urgent & $644(22.6)$ & $636(21.6)$ & $683(23.2)$ & $800(27.2)$ \\
\hline Routine/transplant/unknown & $1,089(38.2)$ & $1,160(39.4)$ & $1,029(34.9)$ & $977(33.2)$ \\
\hline
\end{tabular}

Abbreviation: IQR, interquartile range.

The United Kingdom National Institute for Health and Care Excellence guidelines recommend delirium assessment and intervention by a multidisciplinary team within 24 hours of hospital admission for those at risk. ${ }^{25}$ These guidelines are based on evidence accumulated in clinical studies over the past 30 years suggesting that multicomponent interventions reduce incident delirium by $30 \%$ to $40 \%$ among medical and surgical patients. ${ }^{12,13,25,28}$

Although multicomponent delirium care pathways are associated with improved patient outcomes, the specific clinical benefits might vary across patient populations. Here, we found larger reductions in LOS and total direct cost among medicine patients. Medical patients might respond more robustly to nonpharmacologic multicomponent delirium interventions because of differing delirium etiologies (eg, constipation and sleep deprivation in a medical patient vs seizures or encephalitis in a neurosciences patient). Another explanation for the difference observed in total direct cost might be the inclusion of surgical units in the total study population. For example, not all hospital days are equivalent in cost for patients on a surgical unit.29 For patients requiring surgical care, most of the hospitalization cost might be in- curred during the initial days of hospitalization, when there are perioperative costs; therefore, reduced LOS might have a lower economic impact. ${ }^{29}$

Multicomponent, nonpharmacologic delirium interventions encourage discontinuing restraints. As a result, one might expect a need for more frequent safety attendant use and an associated cost increase. However, we found that the estimated unit-specific values for safety attendant use showed reductions, which were large and highly statistically significant. For all units combined and the medicine unit alone, we found that the rate of restraint use decreased, although the change was not statistically significant. It is possible that some of the interventions taught to nurses and physicians as part of care pathway implementation, such as the use of family support for at-risk and delirious patients, led to a reduction in both safety attendants and restraints.

Our study had several strengths. This is one of the largest hospital-based delirium interventions studied, both in terms of its scope across seven diverse medical and surgical hospital units and the number of hospitalized patients studied. This intervention did not require additional staff or creating a specialized ward. Adherence to the pathway, as measured by risk 
TABLE 3. Unadjusted and Adjusted Clinical Outcomes for All Patients Combined and Medicine Unit Patients

\begin{tabular}{|c|c|c|c|c|}
\hline Clinical outcome & Unadjusted model result $(95 \% \mathrm{Cl})$ & $P$ value & Adjusted model result $(95 \% \mathrm{Cl})$ & $P$ value \\
\hline \multicolumn{5}{|l|}{ All patients } \\
\hline Length of stay proportional change ${ }^{a}$ & $1.00(0.97-1.05)$ & .65 & $0.98(0.92-0.99)$ & .0087 \\
\hline Total direct cost proportional change ${ }^{a}$ & $0.98(0.96-1.00)$ & .17 & $0.99(0.97-1.01)$ & .12 \\
\hline 30-Day hospital readmission odds ratio & $0.93(0.86-1.00)$ & .039 & $0.86(0.80-0.93)$ & .0002 \\
\hline Restraint rate ratio & $0.83(0.76-0.91)$ & $<.0001$ & $0.91(0.71-1.16)$ & .45 \\
\hline Safety attendant rate ratio & $0.51(0.48-0.54)$ & $<.0001$ & $0.63(0.41-0.97)$ & .034 \\
\hline \multicolumn{5}{|l|}{ Medicine unit } \\
\hline Length of stay proportional change & $0.92(0.88-0.95)$ & $<.0001$ & $0.91(0.83-0.99)$ & .028 \\
\hline Total direct cost proportional change & $0.87(0.83-0.91)$ & $<.0001$ & $0.93(0.89-0.96)$ & .0002 \\
\hline 30-day hospital readmission odds ratio & $0.85(0.74-0.98)$ & .029 & $0.97(0.83-1.13)$ & .66 \\
\hline Restraint rate ratio & $1.04(0.89-1.22)$ & .62 & $1.02(0.63-1.67)$ & .92 \\
\hline Safety attendant rate ratio & $0.88(0.80-0.97)$ & .0089 & $0.85(0.68-1.07)$ & .16 \\
\hline
\end{tabular}

a Back-transformed coefficient for length of stay and total direct cost represent proportional change from preintervention to postintervention periods based on log-transformed variables.

assessment and delirium screening, was high (>90\%) 3 months after implementation. This allowed for robust outcome ascertainment. The patient population's characteristics and rates of delirium were stable over time. Because different hospital units incorporated the multicomponent delirium care pathway at different times, limiting enrollment to patients admitted and discharged from the same unit isolated the analysis to patients exposed to the pathway on each unit. This design also limited potential influence of other hospital quality improvement projects that might have occurred at the same time.

The primary limitation of this study is that screening for delirium was introduced as part of the multicomponent intervention. This decision was made to maximize buy-in from bedside nurses performing delirium screening because this addition to their workflow was explicitly linked to delirium prevention and management measures. Delirium could not be ascertained preintervention from the EMR because it is a clinical diagnosis and is coded inadequately. ${ }^{30}$ We could only measure the change in delirium metrics after implementation of the delirium care pathway. Because baseline delirium rates before the intervention were not measured systematically, conclusions about the intervention's association with delirium metrics are limited. All other outcomes were measured before and after the intervention.

Although the comprehensive delirium screening program and high rate of adherence are a methodologic strength of this study, a second limitation is the use of the NuDESC. Our previous research demonstrated that the NuDESC has low sensitivity but high specificity and positive predictive value, ${ }^{26}$ which might underestimate delirium rates in this study. However, any underestimation should be stable over time and temporal trends should remain meaningful. This could allow more widespread study of delirium among hospitalized individuals. Because this care pathway was hospital-wide, it was important to ensure both consistency of screening and longevity of the initiative, and it was necessary to select a delirium assessment tool that was efficient and validated for nursing implementation. For these reasons, the NuDESC was an appropriate choice.

It is possible that our results could be influenced by unmeasured confounders. For example, although we incorporated Elixhauser medical comorbidities and illness severity into our model, we were unable to adjust for baseline functional status or frailty. Baseline functional status and frailty were not reliably recorded in the EMR, although these are potential confounders when investigating clinical outcomes including hospital readmission.

\section{CONCLUSION}

Implementation of a systematic, hospital-wide multicomponent delirium care pathway is associated with reductions in hospital LOS and 30-day readmission. In general medicine units, the reduction in LOS and associated cost savings were robust. These results demonstrate the feasibility and effectiveness of implementing an interprofessional, multidisciplinary multicomponent delirium care pathway through medical center funding to benefit patients and the hospital system.

\section{Acknowledgments}

The authors thank the many hospital staff members, especially the nurses, pharmacists, therapists, and patient care assistants, who helped implement the multicomponent delirium care pathway. All persons who have contributed significantly to this work are listed as authors of this work.

Disclosures: Dr Josephson receives compensation as the JAMA Neurology Editor-in-Chief and Continuum Audio Associate Editor; Dr Douglas received compensation as The Neurohospitalist Editor-in-Chief. The other authors report no disclosures.

Funding: This study was funded by the Sara \& Evan Williams Foundation Endowed Neurohospitalist Chair (Dr Douglas) and the UCSF Clinical \& Translational Science Institute (Dr LaHue) 


\section{References}

1. Bidwell J. Interventions for preventing delirium in hospitalized non-ICU patients: A Cochrane review summary. Int J Nurs Stud. 2017;70:142-143. https://doi.org/ 10.1016/j.ijnurstu.2016.11.010

2. Maldonado JR. Delirium in the acute care setting: characteristics, diagnosis and treatment. Crit Care Clin. 2008;24(4):657-722, vii. https://doi. org/10.1016/j.ccc.2008.05.008

3. Field RR, Wall MH. Delirium: past, present, and future. Semin Cardiothorac Vasc Anesth. 2013;17(3):170-179. https://doi.org/10.1177/1089253213476957

4. Oh ST, Park JY. Postoperative delirium. Korean J Anesthesiol. 2019;72(1):4 12. https://doi.org/10.4097/kja.d.18.00073.1

5. Francis J, Martin D, Kapoor WN. A prospective study of delirium in hospitalized elderly. JAMA. 1990;263(8):1097-1101.

6. Salluh JI, Soares M, Teles JM, et al. Delirium epidemiology in critical care (DECCA): an international study. Crit Care. 2010;14(6):R210. https://doi. org/10.1186/cc9333

7. Ely EW, Shintani A, Truman B, et al. Delirium as a predictor of mortality in mechanically ventilated patients in the intensive care unit. JAMA 2004;291(14):1753-1762. https://doi.org/

8. McCusker J, Cole MG, Dendukuri N, Belzile E. Does delirium increase hospital stay? J Am Geriatr Soc. 2003;51(11):1539-1546. https://doi.org/10.1001/ jama.291.14.1753

9. Inouye SK, Rushing JT, Foreman MD, Palmer RM, Pompei P. Does delirium contribute to poor hospital outcomes? A three-site epidemiologic study. J Gen Intern Med. 1998;13(4):234-242. https://doi.org/10.1046/j.1525-1497. 1998.00073.x

10. Siddiqi N, House AO, Holmes JD. Occurrence and outcome of delirium in medical in-patients: a systematic literature review. Age Ageing 2006;35(4):350-364. https://doi.org/10.1093/ageing/afl005

11. LaHue SC, Douglas VC, Kuo T, et al. Association between inpatient delirium and hospital readmission in patients $>/=65$ years of age: a retrospective cohort study. J Hosp Med. 2019;14(4):201-206. https://doi.org/10.12788/ jhm.3130

12. Hshieh TT, Yue J, Oh E, et al. Effectiveness of multicomponent nonpharmacological delirium interventions: a meta-analysis. JAMA Intern Med. 2015;175(4):512-520. https://doi.org/10.1001/jamainternmed.2014.7779

13. Inouye SK, Bogardus ST, Jr., Charpentier PA, et al. A multicomponent intervention to prevent delirium in hospitalized older patients. N Engl J Med. 1999;340(9):669-676. https://doi.org/10.1056/NEJM199903043400901

14. Marcantonio ER, Flacker JM, Wright RJ, Resnick NM. Reducing delirium after hip fracture: a randomized trial. J Am Geriatr Soc. 2001;49(5):516-522. https:// doi.org/

15. Alhaidari AA, Allen-Narker RA. An evolving approach to delirium: A mixed-methods process evaluation of a hospital-wide delirium program in New Zealand. Australas J Ageing. 2017. https://doi.org/10.1046/j.15325415.2001.49108.x

16. Holroyd-Leduc JM, Khandwala F, Sink KM. How can delirium best be prevented and managed in older patients in hospital? CMAJ. 2010;182(5):465470. https://doi.org/10.1503/cmaj.080519

17. Siddiqi N, Stockdale R, Britton AM, Holmes J. Interventions for preventing delirium in hospitalised patients. Cochrane Database Syst Rev. 2007(2):CD005563. https://doi.org/ 10.1002/14651858.CD005563.pub2

18. Siddiqi N, Harrison JK, Clegg A, et al. Interventions for preventing delirium in hospitalised non-ICU patients. Cochrane Database Syst Rev. 2016;3:CD005563. https://doi.org/10.1002/14651858.CD005563.pub3

19. Inouye SK, Westendorp RG, Saczynski JS. Delirium in elderly people. Lancet. 2014;383(9920):911-922. https://doi.org/10.1016/S0140-6736 (13)60688-1

20. Inouye SK, Charpentier PA. Precipitating factors for delirium in hospitalized elderly persons. Predictive model and interrelationship with baseline vulnerability. JAMA. 1996;275(11):852-857.

21. LaHue SC, Liu VX. Loud and clear: sensory impairment, delirium, and functional recovery in critical illness. Am J Respir Crit Care Med. 2016;194(3):252-253. https://doi.org/10.1164/rccm.201602-0372ED

22. Ritter SRF, Cardoso AF, Lins MMP, Zoccoli TLV, Freitas MPD, Camargos EF Underdiagnosis of delirium in the elderly in acute care hospital settings: lessons not learned. Psychogeriatrics. 2018;18(4):268-275. https://doi.org/10.1111/ psyg. 12324
23. Douglas VC, Hessler CS, Dhaliwal G, et al. The AWOL tool: derivation and validation of a delirium prediction rule. J Hosp Med. 2013;8(9):493-499. https://doi.org/10.1002/jhm.2062

24. Tombaugh TN, McDowell I, Kristjansson B, Hubley AM. Mini-Mental State Examination (MMSE) and the modified MMSE (3MS): A psychometric comparison and normative data. Psychol Assessment. 1996;8(1):48-59. https://doi.org/10.1037/1040-3590.8.1.48

25. Young J, Murthy L, Westby M, Akunne A, O'Mahony R, Guideline Development Group. Diagnosis, prevention, and management of delirium: summary of NICE guidance. BMJ. 2010;341:c3704. https://doi.org/10.1136/ bmj.c3704

26. Hargrave A, Bastiaens J, Bourgeois JA, et al. Validation of a nurse-based delirium-screening tool for hospitalized patients. Psychosomatics. 2017; 58(6):594-603. https://doi.org/10.1016/j.psym.2017.05.005

27. Ely EW, Inouye SK, Bernard GR, et al. Delirium in mechanically ventilated patients: validity and reliability of the confusion assessment method for the intensive care unit (CAM-ICU). JAMA. 2001;286(21):2703-2710. https://doi. org/10.1001/jama.286.21.2703

28. Strijbos MJ, Steunenberg B, van der Mast RC, Inouye SK, Schuurmans MJ. Design and methods of the Hospital Elder Life Program (HELP), a multicomponent targeted intervention to prevent delirium in hospitalized older patients: efficacy and cost-effectiveness in Dutch health care. BMC Geriatr. 2013;13:78. https://doi.org/10.1186/1471-2318-13-78

29. Taheri PA, Butz DA, Greenfield LJ. Length of stay has minimal impact on the cost of hospital admission. J Am Coll Surg. 2000;191(2):123-130. https://doi. org/10.1016/s1072-7515(00)00352-5

30. Fong TG, Tulebaev SR, Inouye SK. Delirium in elderly adults: diagnosis, prevention and treatment. Nat Rev Neurol. 2009;5(4):210-220. https://doi. org/10.1038/nrneurol.2009.24 